\title{
QUAND LES MIGRATIONS DE RETOUR DEVIEN- NENT RÉALITÉ. UN EXEMPLE AVEC LE RETOUR DES PÉRUVIENS
}

\author{
WHEN RETURN MIGRATIONS BECOME A REALITY. THE \\ EXAMPLE OF PERUVIANS WHO RETURN HOME
}

\section{Cuando las migraciones de retorno se transforman en realidad. Un ejemplo con el retorno de los peruanos}

\author{
BRUNO LAFFORT \\ Université de Bourgogne-Franche-Comté - UFR « Sciences du langage \\ de l'homme et de la société ».brunolaffort@hotmail.com
}

Recibido: $13 / 11 / 2015$

Aceptado: 22/12/2015

\begin{abstract}
This paper addresses the issue of "return migrations," which have taken on a new dimension with the crisis that has durably taken root in certain countries of "Old Europe," particularly in Spain. It attempts firstly to shed light on why these migrations have hitherto been understudied, except in a "metaphorical" manner. After presenting a critique of certain notions, in fashion in studies of current migrations, which excessively valorize the "mobility" of migrants, the paper shows that this mobility remains essentially something that the migrants suffer and not choose. Finally, it brings to light the issues involved in return migrations, with the example of Peruvians, based upon field research conducted in Peru in July and August 2014.
\end{abstract}

KEYWORDS: Return immigration, Mobility, Spain, Peru.

RÉSUMÉ: Il s'agit dans cette contribution d'aborder les « migrations de retour », ces dernières prenant une ampleur nouvelle avec la crise qui s'installe durablement dans certains pays de la « vieille Europe », en Espagne notamment. Nous tenterons de comprendre, dans un premier temps, pourquoi ces migrations ont été jusque-là peu étudiées, sauf de manière «métaphorique ». Après une critique de certaines notions en vogue dans l'étude des migrations actuelles valorisant de façon excessive la « mobilité » des migrants, nous montrerons que cette mobilité reste essentiellement subie par ces derniers. Enfin, nous donnerons corps aux migrations de retour avec l'exemple des Péruviens, à partir d'une enquête de terrain réalisée au Pérou en Juillet et Août 2014.

MOTS-CLÉS : Immigration de retour, Mobilité, Espagne, Pérou. 
RESUMEN: Esta contribución aborda las « migraciones de retorno », que han cobrado una gran importancia con la persistente crisis económica que se ha establecido en la "vieja Europa" y, en particular, en España. Se tratará de explicar, en un primer momento, por qué estas migraciones han sido poco estudiadas hasta ahora, salvo de forma "metafórica". Tras realizar una crítica de ciertas nociones que están en boga en el estudio de las migraciones en la actualidad, que ponen en valor de forma excesiva la movilidad de los migrantes, se demostrará que esta movilidad sigue siendo padecida por estos últimos. Por último, se pondrá cara a las migraciones de retorno con el ejemplo de los peruanos, a partir de las entrevistas de campo realizadas en Perú en julio y agosto de 2014.

PALABRAS CLAVE: Inmigración de retorno, Movilidad, España, Perú.

Les migrations de retour constituent, sinon un point noir de la sociologie de l'immigration - tant ces dernières ont été peu abordées par la discipline - du moins un sujet qui n'a été étudié jusque-là que de manière très partielle. Les rares fois où ces dernières ont fait l'objet de recherches empiriques, il s'agit dans presque tous les cas des retours dits «forcés », c'est-à-dire envisagés de manière collective, souvent de façon brutale et non choisie ; un numéro de la Revue européenne des migrations internationales paru en $2013\left(n^{\circ} 3\right)$ intitulé « Migrations de retour et rapatriement » accrédite et développe cette thèse. Ainsi, comme le précise Yann Scioldo-Zürcher dans 1'éditorial, « ce numéro propose d'aborder la notion de rapatriement dans la longue histoire des États qui, à partir de l'époque moderne, ont cherché à assurer la protection de leurs ressortissants ». Et cet auteur de préciser quelques lignes plus loin que « ce numéro fait aussi une large place à l'étude des Français rapatriés d'Algérie ». À notre connaissance, un des rares projets qui a tenté d'aborder ce sujet de manière large reste la publication coordonnée par Véronique Petit pour le compte de la CEPED ${ }^{1}$ et publié en $2007^{2}$. Néanmoins, dans cette étude, la quasi-totalité des contributions abordent les retours de manière métaphorique : l'essentiel de ces dernières ont davantage à voir avec l'identité des uns et des autres plus qu'avec un retour en bonne et due forme, prévu ou tout au moins envisagé dans la durée. Au-delà du fait que ces retours soient difficiles à sérier, cette étude montre finalement que les « vrais » retours au pays, entendus de manière physique et basés sur un acte volontaire, restaient jusque-là soit confidentiels, soit très peu analysés par les sociologues.

\footnotetext{
${ }^{1}$ Le CEPED associe l'Institut National d'Études Démographiques (INED), l'Institut de Recherche pour le Développement (IRD) et l'Université de Paris 5. Il consacre ses activités à stimuler la collaboration scientifique entre des équipes de recherche du Nord et du Sud dans le domaine de la population et du développement.
}

${ }^{2}$ PETIT, V. (2007). Migrations internationales de retour et pays d'origine, Nogent-sur-Marne. 
On pourrait dans un premier temps réfléchir sur le faible nombre de ces études. Tout d'abord, en dehors des retours forcés, il est vrai que les personnes qui décident, de manière individuelle et en leur « âme et conscience » - c'est-àdire de façon volontaire -, de rentrer dans leur pays d'origine après plusieurs années passées dans un pays tiers (le « pays d'accueil ») ne sont pas légion. Toutes les enquêtes montrent en effet que malgré moult difficultés rencontrées dans le pays d'accueil, les personnes rechignent à " rentrer au pays » tant elles craignent le coût symbolique d'une nouvelle réadaptation. Au niveau des sociologues eux-mêmes qui travaillent sur les migrations, il est aussi possible de pointer un certain ethnocentrisme, ces derniers voulant se persuader que leur pays reste malgré tout « accueillant», voire bienveillant avec l'Étranger, et que de tels retours restent, dès lors, inenvisageables ou du moins très marginaux. Mais au delà de l'hospitalité et de l'accueil - terme que nous définirons comme le versant institutionnalisé de l'hospitalité -, nous verrons que la possibilité ou pas, de continuer à exercer un travail décent reste un critère déterminant pour « franchir le pas » du retour. En ce sens, rappelons que l'origine de toute migration réside dans la quête d'un avenir meilleur. Enfin, il subsiste sans nul doute le poids du père fondateur de la discipline, Abdelmalek Sayad. Celui-ci a montré que le retour au pays se vivait quasiment toujours sur le mode du renoncement, voire de la honte ; à ce titre, le retour reste éminemment un mythe, et s'il ne constitue pas un impensé, sa mise en œuvre très concrète demeure la plupart du temps impensable. C'est aussi ce que nous avons montré dans quelques-unes de nos recherches ${ }^{3}$.

Toutes ces tentatives d'explication restent fondées et c'est sans doute ce faisceau d'éléments qui permet de comprendre le faible nombre des études actuelles. Pour autant, aujourd'hui, ces migrations de retour individuelles existent bel et bien, même s'il est difficile d'en connaître précisément le " poids » d'un strict point de vue quantitatif. Elles concernent essentiellement des personnes présentes dans quelques pays de la vieille Europe ayant opéré une véritable mutation - passant en quelques années seulement du statut de pays dits " de départ » à celui dit « d'accueil » - liée à leur développement économique considérable du milieu des années 1990. Nous pensons à l'Irlande, mais aussi - et surtout - à l'Espagne. Ces deux pays ont connu un «boom » économique sans précédent pendant les années 2000 à 2008, essentiellement dû à l'essor d'un capitalisme financier qui se joue désormais, non seulement des frontières, mais aussi des hommes et des femmes qui accompagnent son développement. Avec la crise de 2008, ces deux pays ont vu leur croissance, par trop idyllique, redes-

3 "Le mythe du retour", Hommes et Migrations, 2005, n 1253, janvier-février, pp. 105-116 et Entre ici et là-bas. Des Maghrébins racontent (2014), Paris, Karthala. 
cendre comme un vulgaire soufflé sorti du four. Cette crise s'est traduite par la destruction de milliers d'emploi et a entrainé le départ de dizaines de milliers de personnes dont la plupart sont rentrés dans leur pays. Avant de revenir sur notre sujet, il s'avère nécessaire de définir un peu mieux les différentes acceptions de ces migrations de retour.

\section{MIGRATIONS DE RETOUR : DE QUOI PARLE-T-ON ?}

Nous l'avons dit, les documents sur le sujet restent confidentiels. De plus, l'objet lui-même semble flou et se prête mal à une tentative de définition claire ; mais n'est-ce pas le cas de tous les objets étudiés en sciences humaines ? Le premier consensus souligné par Véronique Petit - coordinatrice de l'ouvrage évoqué (op. cit.) - dans sa présentation nous interpelle. Il semblerait ainsi que l'étude des retours en tant que tels ne soit plus pertinente, tant « la notion de retour renvoie à un découpage de la circulation migratoire, à une séquence de déplacement que l'observateur extrait d'une contexte et d'une dynamique, alors que dans la pratique des individus le retour est davantage pensé et vécu comme une étape, un moment dans la trajectoire migratoire, et non comme une fin en $\operatorname{soi}^{4} »$.

Circulation migratoire : voici un terme utilisé par l'auteur devenu presque un « incontournable » de la sociologie actuelle des migrations. Ainsi, dans une approche dynamique, il s'agirait désormais de privilégier les « trajectoires » et les « circulations » des migrants, tout cela au détriment d'une sociologie plus classique qui se basait sur une approche plus déterministe. Certes, cette notion de mobilité ne doit pas être occultée. Ainsi, Véronique Petit nous conduit à « décentrer notre regard sur la mobilité, regard qui pendant des années a été centré sur le pays d'arrivée » (Ibid., p. 10). Les recherches de Emmanuel Ma Mung et de Alain Tarrius ont souligné, à juste titre, le versant dynamique des migrations, quelque peut absent des analyses déterministes initiées par Abdelmalek Sayad. Mais cette focalisation - parfois excessive - sur les trajectoires, ne risque-t-elle pas de cautionner un capitalisme financier de plus en plus brutal qui se joue aujourd'hui des personnes comme des marchandises? Dans une vision un peu similaire, on trouve les analyses de Sonia Parella Rubio, Alisa Petroff et Olga Serradell Pumareda. Ces trois auteurs se réfèrent, en les reprenant, aux travaux de Nina Glick Chiller et al. qui développent de manière positive la perspective transnationale dans l'étude des migrations ainsi que les études sur la mobilité, deux notions qui

${ }^{4}$ PETIT, V., ibid., p. 13. 
incluaient de facto "le retour comme partie du cycle migratoire $»^{5}$. Cette mobilité, ultime ironie de l'histoire des migrations, en incluant en quelque sorte le retour des immigrés dans leur pays comme faisant partie intégrante d'un processus, d'un « cycle », permettrait de cautionner les vertus de ce capitalisme financier.

Pour le dire autrement, le vieux débat qui traverse la sociologie - entre déterminisme absolu et liberté individuelle - semble aujourd'hui pencher, au niveau de la sociologie des migrations tout au moins, du côté de l'autonomie des acteurs. Comme l'écrit Emmanuel Ma Mung', « il s'agit de mettre l'accent sur les initiatives plutôt que sur les déterminismes et d'étudier la façon dont les individus et les groupes aménagement pour eux-mêmes, les "conditions extérieures". Ce chercheur définit dans cette même contribution la notion de projet migratoire qui permettrait d'inclure une dimension explicative médiane dans le " choix » migratoire : le rôle joué par la famille. Mais peut-on affirmer que chaque migrant, lorsqu'il quitte son pays, dispose d'un véritable « projet migratoire » ? Nos récentes enquêtes de terrain ne confortent pas toujours ce postulat.

En d'autres termes, s'il est indéniable que le migrant circule, certes, on peut aussi penser qu'un jour il souhaitera, comme tout un chacun, « poser ses valises » quelque part et s'installer durablement dans un lieu, physiquement, en optant pour un pays bien réel. Une focalisation excessive sur les mobilités reviendrait à déplacer le « balancier sociologique » vers une survalorisation du « jeu des acteurs", en faisant fi des déterminismes dont on connaît l'importance, notamment dans ce champ de la sociologie de l'immigration (Sayad, 1991 et 1999). Cette « conception intégrée » du retour pourrait s'interpréter aussi comme une manière, pour le pays d'accueil, de se défausser de ses responsabilités eu égard à son « impossible accueil » sur le long terme des personnes ayant pourtant grandement contribué à son développement économique. Ainsi, les thèmes qui semblent faire l'objet d'un quasi-consensus au sein de la discipline doivent être réinterrogés ; en sociologie de l'immigration, ces derniers se nomment aujourd'hui «mobilité », « transnationalisme » et « circulation migratoire ».

Un des sociologues ayant largement contribué à développer cette notion de mobilité, Alain Tarrius, un temps tenté par cette survalorisation des initiatives de migrants qui se joueraient des frontières, a bien compris que ces mobilités

\footnotetext{
${ }^{5}$ PARELla RUBiO, S., PETROFF, A. y SERRAdEll PUMAREDA, O. (2014), "Programas de retorno voluntario en Bolivia y España en contextos de crisis", in Sassone S. y Yépez del Castillo I. (coord.), Migración y crisis global. Europa-America latine: nuevas estrategias, nuevas desigualdades, Revista CIDOB d'Afers Internacionals, n 106-107, septiembre, p.172.

${ }^{6}$ MA MUNG, E. (2009), "Le point de vue de l'autonomie dans l'étude des migrations internationales: "penser de l'intérieur" les phénomènes de mobilité", in Dureau F. et Hily M.-A., Les mondes de la mobilité, Presses de l’Université de Rennes, Rennes, p. 28.
} 
étaient largement imposées par la globalisation ; son dernier ouvrage qui tente une comparaison entre les migrants pauvres et les jeunes résidants dans les banlieues françaises le confirme : « Les migrants internationaux pauvres ont compris le refus d'hospitalité des nations riches. Un grand nombre d'entre eux - autour de 200000 annuellement pour la France, 600000 pour l'Europe - ne se présentent plus à nous comme é- ou immigrants, mais comme transmigrants. En perpétuel mouvement entre nations, ils sont devenus les colporteurs du capitalisme marchand moderne ${ }^{7}$. » La mobilité renvoie davantage à une contrainte qu'à un choix supposé d'individus en quête de liberté et d'autonomie.

Avant de se centrer plus avant le retour des Latino-Américains - et notamment des Péruviens sur lesquels nous allons revenir dans cette contribution -, essayons d'esquisser les types de retour possible. Il existe déjà les déplacements quasiment forcés (ou rapatriements), comme ceux des « Pieds-noirs » d'Algérie après l'indépendance de ce pays ${ }^{8}$. Mais s'agit-il véritablement d'un " retour » quand on sait que la plupart des ces Pieds-noirs ne connaissaient pas la France ? Le retour des Harkis, Algériens ayant combattus dans l'armée française, soulève là encore des questions sémantiques, la plupart de ces Algériens n'ayant, là non plus, jamais connu ce pays auparavant. Autres retours évoqués dans la littérature : celui des Juifs qui retournent en Israël (l'Aliyah) sans connaître, là non plus, physiquement ce pays. Celui des retraités - notamment des Maghrébins ayant travaillé en Europe - se rapproche un peu de nos préoccupations car il s'agit ici d'un retour physique de personnes ayant quittées leur pays dans leur jeunesse. Enfin, les migrations de retour « internes » au sein d'un même pays ont été peu étudiées, à l'exception notable de Teofilo Altamirano, sociologue péruvien. Dans une étude réalisée en 1985, il s'agissait pour lui de comprendre comment les personnes venant de la campagne péruvienne et attirées un temps par les « lumières de la ville » en étaient venues à faire machine arrière, par une ville vécue comme peu accueillante et ne leur garantissant ni un logement décent, ni un travail moins précaire que celui qu'il exerçaient précédemment dans l'agriculture.

Dans ces exemples de retour, deux thématiques reviennent : le retour mythifié d'un côté (qui peut s'entendre jusqu'à l'étude du « retour » des enfants de migrants), et les allers-retours des personnes (souvent retraitées, mais pas uniquement) de l'autre. Comme le souligne Véronique Petit (op. cit.) à propos des enfants de migrants, leur décision peut s'interpréter « comme un retour aux origines ou comme un retour au pays des parents ou des aïeux. Du point de

\footnotetext{
7 TARRIUS, A., MISSAOUI L. et QACHA, F. (2013), Transmigrants et nouveaux étrangers : hospitalités croisées entre jeunes des quartiers enclavés et nouveaux migrants internationaux, Toulouse, Presses universitaires du Mirail, extrait de la quatrième de couverture.

${ }^{8}$ Notons néanmoins qu'une minorité de Français d'Algérie n'ayant pas pris part à la guerre, et souvent de condition sociale modeste, ont choisi de rester en Algérie.
} 
vue de l'observation stricte de la mobilité, il n'y a pas de retour, c'est-à-dire un déplacement précédé d'un aller ${ }^{9} \gg$. Le retour des enfants d'immigrés dans un pays - celui de leurs parents - qu'ils n'ont pas connu, conserve étonnement le vocable de « retour » comme le montre Fanny Jedlicki à propos du retour au Chili des enfants d'exilés chiliens, qui se nomment - et sont nommés - retornados ${ }^{10}$. Quant aux nombreux allers-retours, ces derniers sont extrêmement vivaces, par exemple entre la France et la Maghreb, le développement des compagnies aériennes low coast ayant accru de manière considérable ces derniers. De manière plus imagée, voire métaphorique, certains évoquent enfin le retour virtuel, via les transferts financiers, dont on sait qu'ils sont extrêmement conséquents entre des pays comme la France et le Maroc ${ }^{11}$. Bref, qu'il s'agisse d'un retour symbolique, d'un retour dans une terre physiquement inconnue ou encore du « dernier retour» (pour le rapatriement du corps du défunt dans son pays natal), on se trouve en face d'une pléthore de situations qu'il s'avère difficile de comparer entre elles.

Comment tenter de cerner plus précisément le retour sur lequel nous allons travailler dans cet article ? Sonia Parella Rubio et al. proposent une première approche par défaut de ce « retour volontaire » (définition tirée d'un travail menée par le Réseau européen des migrations en 2009), en pointant tout ce qu'il n'est pas : " cette conception du retour exclue les processus de déportation, d'expulsion, de rapatriement et d'extradition, tous ces procédés ne prenant pas en compte l'élément volontaire comme élément de définition ${ }^{12}$. » Nous proposons, pour notre part, la définition suivante : il s'agit d'un retours volontaire, non forcé et opéré de manière individuelle, dans le pays d'origine habité précédemment, de personnes en âge de travailler.

\section{CARACTÉRISTIQUES DE L'IMMIGRATION RÉCENTE EN ESPAGNE}

Avant de sérier quelques éléments permettant à certaines personnes d'avoir mis en œuvre le retour, nous devons dégager quelques caractéristiques de l'immigration présente en Espagne, avant d'aborder celle des Latino-Américains, puis des Péruviens. Cette immigration récente a indéniablement accompagné l'essor du capitalisme financier, comme nous l'avons déjà évoqué ; la croissance extrêmement rapide de l'Espagne n'a pu se dérouler que grâce à l'apport d'une main d'œuvre étrangère conséquente. Une première enquête que nous avons pu

\footnotetext{
${ }^{9}$ PETIT, V., op. cit., p.13.

${ }^{10}$ Migrations de retour et pays d'origine, op. cit., p. 197.

${ }^{11}$ CHAREF Mohamed, Migrations de retour et pays d'origine, ibid., p. 129.

${ }^{12}$ PARELLA et al., op. cit., p. 174.
} 
mener lors de la crise de 2008, en Andalousie, nous a dévoilé ces mécanismes dans toute leur tragédie, lorsque le travail était venu à manquer, laissant dans le dénuement le plus complet des milliers de Subsahariens venus chercher un travail dans l'agriculture. L'Espagne a donc connu en quelques années une croissance de population étrangère fulgurante. En 1998, on y dénombrait seulement 600000 étrangers et en 2007 4,5 millions. C'est aujourd'hui le premier pays de l'UE où la moyenne des entrées annuelles avoisine les 600000 personnes. Le deuxième pays de l'Union en termes d'arrivée est l'Italie, avec 400000 entrés annuelles. Les autres pays où l'immigration reste conséquente - le Royaume-Uni, la France et l'Allemagne - sont loin derrière avec seulement 200000 entrées par an. Les Marocains constituent ce que l'on pourrait appeler l'immigration « historique », avec une population d'environ $783000^{13}$ personnes actuellement. Les Roumains, avec une immigration n'ayant véritablement débutée qu'au début des années 2000, ont supplanté cette immigration historique pour devenir la première communauté étrangère, avec près de 896000 individus. Enfin, les Équatoriens représentent la quatrième nationalité étrangère en Espagne (après les Anglais), avec un peu plus de 306400 personnes. Les Subsahariens n'apparaissent dans les statistiques que ne manière très marginale alors que ce sont eux qui constituent l'immigration la plus récente, notamment par le nombre de personnes arrivées de manière clandestine.

L'analyse de la pyramide des âges des Roumains et des Latino-Américains montre que ces dernières sont plus « équilibrées » que celle des Marocains, avec une présence de femmes autant que d'hommes. Le caractère familial de ces deux colectivos arrivés davantage en famille ${ }^{14}$ marque une inflexion par rapport aux Marocains, venus seuls, de manière plus conforme aux schémas de l'immigration dite " classique ». Enfin, les immigrés se retrouvent concentrés dans un petit nombre de régions : la Catalogne (pour son industrie), la région de Madrid (pour l'attraction de la capitale), la région de Valencia (pour le bâtiment et l'agriculture) et enfin celles de Murcia et de l'Andalousie (pour l'agriculture). Comment les Espagnols évoquent les personnes étrangères présentes sur leur territoire ? Inmigrantes semble être le terme générique par lequel ces derniers désignent les étrangers venus travailler dans leur pays. Mais à y regarder de plus près, ce terme charrie avec lui une connotation péjorative ; de plus, il fait implicitement référence aux Maghrébins, aux Subsahariens, aux Roumains et dans une moindre mesure aux Latino-Américains. Le vocable d'extranjero semble être plutôt réservé

\footnotetext{
${ }^{13}$ OCDE (2013), Perspectives des migrations internationales, Paris, OECD iLibrary, juin, p. 399, pour ces chiffre et les suivants dans ces deux paragraphes.

${ }^{14}$ Les latino-Américains viennent en premier lieu le plus souvent seuls, mais opèrent le regroupement familial très rapidement.
} 
à l'immigration « blanche ", de villégiature, originaire de l'Union européenne, mais également provenant des pays du Golfe. Il existe en effet une importante immigration « touristique-résidentielle » dans ce pays, représentée essentiellement par les Anglais et les Allemands. Les Britanniques, avec plus de 397500 personnes, représentent en effet la troisième communauté étrangère en Espagne.

Il est indéniable que les immigrés occupent - en Espagne comme dans les autres pays européens - des secteurs très spécifiques de l'économie, appelés par les sociologues espagnols « niches de travail » (nichos laborales). On retrouve donc en Espagne une immigration essentiellement économique, une immigration de travail cantonnée à certains travaux. De tout temps, en Espagne comme en France, en Libye ou ailleurs, les immigrés ont toujours été présents pour assurer et assumer les travaux délaissés par les nationaux, travaux revêtant un caractère à la fois dur physiquement et (ou) dévalorisé par l'ensemble de la société, et généralement très mal payés. Les secteurs où se retrouvent majoritairement les immigrés en Espagne sont ainsi ceux de l'agriculture, du bâtiment et des travaux publics, de la restauration et des services domestiques (notamment envers les personnes les plus âgées), ces derniers étant en pleine explosion dans les pays de la vieille Europe. Pour ces nouveaux emplois de service à la personne, Robert Castel parle « d'emplois de type servile » et de « néo domesticité » parce qu'on se situerait, selon lui, en deçà du rapport salarial moderne avec ces emplois-là. Ce sociologue se demande même si la prolifération de ce type de services ne serait pas un indice de "tiers-mondisation" de nos sociétés "développées". Qu'en est-il de le division sexuée des tâches ? Le secteur de l'agriculture regroupe essentiellement les hommes, sauf dans certaines cultures sous serre (telles que les fraises, dans la région de Huelva) où ce sont les femmes qui dominent ; c'est l'inverse pour le secteur des « aides à la personne » assuré de manière quasi exclusive par les femmes, notamment par les Roumaines et les Latino-Américaines ; dans la restauration, hommes et femmes se retrouvent à part égale. Cette féminisation de l'immigration constitue un premier changement notable avec l'immigration dite des « premiers âges » décrite par Abdelmalek Sayad ; le deuxième changement renvoie à la constitution d'une immigration familiale où chaque personne au sein du couple va tenter de trouver un emploi.

José Menor Toribio (Université de Jaén) tente d'analyser, tout d'abord, la mobilité de ces immigrés en Espagne. Il compare leur « destins primaires » - c'est-à-dire leur premier lieu d'arrivée en Espagne - avec leur « destin secondaire » - constituant leur lieu actuel de résidence - grâce aux statistiques de l'INE (Instituto National de Estadistica) élaborés à partir de la population étrangère empadronada, c'est-à-dire enregistrée dans la commune où elle réside. Son analyse permet de mettre en évidence « la grande mobilité de la population étrangère qui pour l'année 2006 fut quatre fois supérieure à celle de la population espagno- 
$1 \mathrm{e}^{15} »$. D'autres chercheurs, à l'instar de Cohen et al., ont confirmé cette tendance en montrant justement que la moitié des personnes étrangères ne résident plus dans leur premier domicile déclaré.

Cette extrême mobilité - et par là même précarité - s'est développée par la conjonction de deux éléments. Le premier provient d'un marché du travail extrêmement libéral, où les contrats précaires et de courte durée font florès ; le deuxième du développement d'une agriculture intensive qui s'est accrue depuis l'entrée de l'Espagne dans l'UE, agriculture qui requiert néanmoins une main d'œuvre toujours importante durant les périodes de récolte. Cette libéralisation du marché du travail est soulignée par la plupart des économistes espagnols, à l'instar de José Carlos Diez, économiste en chef de Intermoney : « L'Espagne est un des marchés du travail les plus flexibles de la planète. Un tiers des travailleurs ont un contrat temporaire ${ }^{16}$. S'il fallait s'en convaincre encore davantage, écoutons Claudio Boada, le « patron des patrons » espagnol, Président du Cercle des entrepreneurs (l'équivalent du MEDEF français), reconnaître lui-même cette état de fait : « Nous avons flexibilisé le marché du travail d'une manière totalement abusive (...) Qu'avons nous fait ? Nous avons abusé clairement des embauches temporaire ${ }^{17} \gg$ Rappelons enfin que cette volonté de spécialisation à outrance de l'agriculture a été fortement incité par Bruxelles qui souhaitait transformer l'Espagne et l'Italie en « vergers » de l'Europe.

Cette absence de stabilité ne favorise pas l'installation des immigrés et par là même leur intégration. Au risque de nous répéter, la mobilité reste souvent illusoire. En dehors de quelques personnes privilégiées qui pourraient se retrouver dans ces termes-là - nous pensons par exemple aux étudiants étrangers présents en Espagne, dont la plupart viennent de « bonnes familles » - il s'avère plus que nécessaire de souligner que toutes les autres ne choisissent pas cette mobilité, mais la subissent.

\section{LES LATINO-AMÉRICAINS EN ESPAGNE}

Nous mettrons à profit dans cette section le numéro 106-107 de la revue CIDOB d'Afers Internacionals paru en septembre 2014 et intitulé « Migrations et crise globale. Europe-Amérique latine : nouvelles stratégies, nouve-

\footnotetext{
${ }^{15}$ TORIBIO MENOR, J. A. (2009), “Implicaciones territoriales y demográficas de la inmigración : fuentes y análisis", In Molina Navarette, C., Pérez Sola, N. y Esteban de la Rosa, G. (coord.), Inmigración e integración de los extranjeros en España, Madrid: Difusión jurídica, p. 188.

${ }^{16}$ El País, Domingo 21 de diciembre de 2008.

${ }^{17}$ El País, Domingo 21 de diciembre de 2008.
} 
lles inégalités ${ }^{18} »$. Ce numéro est une première tentative pour rendre compte des changements intervenus dans le champ migratoire, tout particulièrement en Espagne, depuis la crise de 2007-2008 ${ }^{19}$. Dans l'introduction, les coordonateurs du numéro, Susana Sassone et Isabel Yépez del Castillo, soulignent que depuis la fin du $\mathrm{XX}^{\text {ème }}$ siècle, ce ne sont plus les États-Unis qui constituent pour les Latino-Américains leur pays de destination principal, mais la Vieille Europe, et notamment l'Espagne : " le capitalisme globalisé, en rendant effective la division du travail, a commencé à attirer [en Espagne, nda] des centaines de milliers de Latino-Américains. » (p. 8). Pour autant, cette nouvelle destination, très prisée, le sera sur un laps de temps très bref puisque ces mêmes auteurs notent qu' "en 2007, le scénario change [avec] l'installation de la crise globale [qui] aura de multiples effets $»^{20}$. Les migrations des Latino-Américains, au niveau de l'ensemble des pays européens, ne sont pas importantes - en chiffre comme en pourcentage - comparées à celles provenant de l'Afrique, du Maghreb et de l'Asie. Néanmoins, elles vont se retrouver essentiellement en Espagne, au Portugal et en Italie - ce qui va augmenter de facto leur représentativité dans ces trois pays. Les plus nombreux à avoir traversé l'Atlantique et tenté l'expérience migratoire sont les Brésiliens, suivis des Équatoriens, des Colombiens, des Argentins, des Péruviens, des Vénézuéliens et enfin des Boliviens.

Concernant l'Espagne, à partir du début des années 2000, si l'on met de côté l'immigration marocaine dont, nous l'avons dit, la présence est plus ancienne, ce sont d'abord les Roumains qui constituent la plus grosse partie des arrivées, même si derniers ont eux aussi, pour partie, regagné la Roumaine depuis la crise. Les Latino-Américains arrivent ensuite, l'essentiel des entrées étant assurées par les Équatoriens et les Colombiens. Notons enfin que les entrées ont été divisées par deux entre 2001 et 2007, ce qui témoigne de l'arrivée, bien réelle, de la crise. Enfin, ce premier tableau corrobore le taux important de féminisation de cette immigration récente, autant chez les Roumains que chez les Latino-Américains, avec l'envolée des emplois dans les services d'aide à la personne.

\footnotetext{
${ }^{18} \mathrm{Ce}$ titre, ainsi que tous les extraits qui suivent émanant de cette revue, sont traduits par nos soins de l'espagnol au français.

${ }^{19}$ Ce numéro reprend des contributions présentées lors du colloque organisé par le Consejo Europeo de Investigaciones Sociales, les 12-15 juillet 2013 à Oporto (Portugal), avec l'aide de l'Université Fernando Pessoa.

${ }^{20}$ SASSONE S. y YÉPEZ DEL CASTILLO I. (coord.), Migración y crisis global. Europa-America latine: nuevas estrategias, nuevas desigualdades, Revista CIDOB d'Afers Internacionals, $\mathrm{n}^{\circ} 106-$ 107, septiembre, p. 14.
} 
Tableau 1 : Entrées des étrangers en Espagne, selon les années et par nationalité

\begin{tabular}{|l|l|l|l|l|l|}
\hline Pays & 2001 & 2004 & 2007 & 2011 & $\begin{array}{l}\% \text { de femmes } \\
\text { en 2011 }\end{array}$ \\
\hline Maroc & 39500 & 73400 & 85000 & 43200 & 42 \\
\hline Roumanie & 23300 & 103600 & 197600 & 60900 & 52 \\
\hline Équateur & 82600 & 17200 & 30200 & 8800 & 49 \\
\hline Colombie & 71200 & 21500 & 41700 & 16100 & 54 \\
\hline Bulgarie & 11800 & 21000 & 31300 & 11900 & 49 \\
\hline Pérou & 7100 & 17700 & 27400 & 9300 & 57 \\
\hline $\begin{array}{l}\text { Royaume-Uni et } \\
\text { Allemagne }\end{array}$ & 20900 & 58300 & 51200 & 25200 & $/$ \\
\hline Total autres pays & 137600 & 333100 & 456100 & 240900 & $/$ \\
\hline Total & 394000 & 645800 & 920500 & 416300 & 49 \\
\hline
\end{tabular}

Source : D’après « Crisis global y el sistema migratorio Europa-America latina », in Migración y crisis global. Europa-America latina : nuevas estrategias, nuevas desigualdades, Susana Sassone et Isabel Yépez del Castillo (coords.), 2014, p. 18

Nous devons néanmoins relativiser le caractère familial de cette immigration, comme Virginie Baby-Collin et Geneviève Cortès nous y invitent. Dans leur article centré sur le retour des Boliviens ${ }^{21}$ dans ce même numéro de la revue CIDOB (op. cit.), ces auteures constatent d'abord que deux tiers des migrants boliviens, hommes et femmes, sont arrivés seuls en Espagne. Elles notent ensuite « la proportion très élevée de familles séparées par la distance ; $36 \%$ des hommes et $21 \%$ des femmes mariés qui vivent en Espagne ne vivent pas avec leur conjoint, et parmi les $32 \%$ qui ont des enfants, plus de la moitié les ont laissés dans leur pays $^{22} \gg$. Il est intéressant de comparer ces entrées annuelles avec les effectifs des étrangers (en stock) présents sur la même période (cf. tableau $\mathrm{n}^{\circ} 2$, ci-dessous) :

Tableau 2 : Effectifs des étrangers par nationalité et par ordre décroissant pour l'année 2011

\begin{tabular}{|l|l|l|l|l|}
\hline Pays & 2001 & 2004 & 2007 & 2011 \\
\hline Roumanie & 67300 & 317400 & 731800 & 896000 \\
\hline Maroc & 307500 & 511300 & 652700 & 783100 \\
\hline
\end{tabular}

\footnotetext{
${ }^{21}$ « Nuevos despliegues del campo migratorio boliviano frente a la crisis », pp. 61-83.

${ }^{22}$ BABY-COLLIN, V. et CORTÈS, G., op. cit., p. 66.
} 


\begin{tabular}{|l|l|l|l|l|}
\hline Pays & 2001 & 2004 & 2007 & 2011 \\
\hline Royaume-Uni & 128100 & 227200 & 353000 & 397500 \\
\hline Équateur & 259500 & 497800 & 427700 & 306400 \\
\hline Colombie & 191000 & 271200 & 284600 & 244700 \\
\hline Allemagne & 113800 & 133600 & 181200 & 196700 \\
\hline Pérou & 44800 & 85000 & 121900 & 121900 \\
\hline Total autres pays & 865900 & 1687100 & 2515900 & 2764700 \\
\hline Total & 1977900 & 3730600 & 5268800 & 5711000 \\
\hline
\end{tabular}

Source : D’après « Crisis global y el sistema migratorio Europa-America latina », op. cit., p.19

Si l'on agglomère ne serait-ce que les Équatoriens, les Colombiens et les Péruviens pour l'année 2011, les Latino-Américains apparaissent aujourd'hui aussi nombreux en Espagne que les Marocains et les Roumains. Parmi tous ces immigrés, ce sont les Latino-Américains qui ont bénéficié - sinon d'un accueil - du moins d'un statut privilégié. Tout d'abord, le gouvernement espagnol a permis à certains colectivos sud-américains de rentrer en Espagne avec des facilités administratives (telles que l'absence de visa), mais aussi d'obtenir un permis de travail, puis de résident. Il faut sans doute y voir une subsistance des liens historiques entre l'Espagne et ses anciennes colonies. Ensuite, la langue commune et la religion catholique (très prégnante en Amérique latine comme en Espagne) ont constitué sans doute des facteurs de facilitation. Francisco Torrez Pérez revient sur cette vision : « de manière générale, les immigrés latino-américains ont connu une insertion tranquille, sans grandes tensions (...) Les Latino-Américains ont bénéficié, par rapport aux autres immigrés, d'une valorisation sociale très positive $^{23}$. » Il faut enfin insister sur la spécialisation extrême des emplois en Espagne, basée sur une véritable politique de quotas (cupos) pour les emplois « non couverts » par les Espagnols et dont la liste est renouvelée chaque année par le gouvernement, cela ayant engendrée une véritable division du travail entre les différents groupes d'inmigrantes.

Les Marocains ont quasiment toujours été cantonnés dans l'agriculture et le bâtiment, ces derniers étant sensés (sic) « se satisfaire de la nécessité d'une main d'oeuvre saisonnière dans l'agriculture ${ }^{24} »$. Les Latino-Américains, quant à eux, se sont « spécialisés » dans le secteur des services et comme employés domesti-

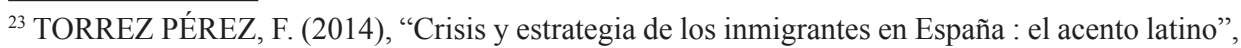
in Sassonne, S.. et Yépez des Castillo, I., op. cit., p. 216.

${ }^{24}$ PAERREGAARD, K. (2013), Peruanos en el mundo. Una etnografía global de la migración, Lima, Fondo Editorial de la Pontificia Universidad Católica del Perú, p. 83. 
que. Ces derniers sont « très présents dans le tertiaire $(70 \%$, soit une part équivalente à celle de l'ensemble de la population, contre $61 \%$ pour l'ensemble des étrangers), et peu dans l'agriculture (qui emploie $22 \%$ des Africains, et moins de $5 \%$ des Latino-américains $)^{25} »$. Les Latino-Américains étaient semble-t-il « préférés aux autres étrangers parce que souvent ils avaient une formation ou une connaissance dans les professions de santé, se comportaient avec respect avec les personnes âgées et avaient des compétences dans la langue espagnole ${ }^{26} »$. Mais depuis le début de la crise, les choses ont notoirement changé, celle-ci ayant déstabilisée ce processus d'insertion socioprofessionnel, des Latino-Américains comme des autres immigrés présents sur le sol espagnol.

Avant d'analyser les possibilités d'actions, somme toute très relatives, des Latino-Américains pour affronter une crise qui perdure, rappelons quelques données de cette dernière. En 2014, l'Espagne comptait fin mars près de 6 millions de chômeurs, soit 26 \% de sa population active ${ }^{27}$. Le chômage des jeunes, avec un taux de 55,5\%, a dépassé celui de la Grèce. Mais au delà de ces taux extrêmement préoccupants, de grosses disparités subsistent entre les natifs et les immigrés. Comme le souligne Francisco Torrez Pérez, « le chômage affecte de forme distincte les travailleurs espagnols, avec un taux de chômage de 24,8 \% au quatrième semestre 2013, et les étrangers avec un taux de $36,6 \%{ }^{28} 》$. Parmi les chômeurs de longue durée, les Latino-Américains représentent $41,5 \%$ des effectifs. Mais là encore, il existe des différences notables entre les différents colectivos. Et ce même chercheur de poursuivre qu' « en 2011, le taux de chômage des Latino-Américains était beaucoup plus élevé que celui des autochtones, 28,5\% contre $19,5 \%$ respectivement, mais il était le plus bas de tous les immigrés extracommunautaires ; ainsi, le taux de chômage des Africains avait grimpé à 49,3\% ».

\subsection{Spécificités de l'immigration péruvienne depuis les années 1990}

La migration des Péruviens fut longtemps cantonnée aux États-Unis, puis dans quelques pays européens (dont la France) où il s'agissait surtout d'étudiants. Ce n'est qu'à partir du début des années 1980 que ces migrations commencent à s'étendre à tous les pays européens, ainsi qu'au Japon. Ceci dit, la majeure partie des Péruviens ayant tenté l'aventure migratoire vivent dans les pays frontaliers (Chili, Bolivie et Équateur) ou proches (Argentine), mais aussi - et surtout - aux États-Unis. À l'instar des Boliviens, les Péruviens ont commencé à « revoir »

\footnotetext{
${ }^{25}$ BABY-COLLIN, V., CORTÈS, G. et MIRET, N. (2009), "Migrants andins en Espagne: ruptures et continuités d'une géographie économique de l'immigration", Méditerranée, n 113, p. 50.

${ }^{26}$ PAERREGAARD K. citant ESCRIVA, op. cit., p. 85.

${ }^{27}$ Le Monde du 29 avril 2014.

${ }^{28}$ TORREZ PÉREZ, F., op. cit., p. 220.
} 
leur pays de destination au début du XXI ${ }^{\text {ème }}$ siècle, avec la crise argentine des années 2000 et le durcissement d'entrée aux Etats-Unis après le 11 septembre 2001. Mais au-delà, d'autres éléments spécifiques ont conduit ces derniers à émigrer : le climat terroriste de l'époque avec les nombreux attentats commis par des groupes se réclamant de l'extrême gauche (le Sentier Lumineux et le Mouvement Révolutionnaire Tupac Amaru) et, un pu plus tard, le deuxième mandat du président Fujimori, gagné par l'autoritarisme et finalement emporté par la corruption. L'histoire des Péruviens et des Japonais est assez singulière pour être rappelée ici. Tout comme le président Fujumori, nombre de Péruviens sont des descendants de migrants japonais arrivés au Pérou entre les années 1899 et 1930, comme le montre les travaux de Takenaka; ces derniers sont identifiés à des nikkeijin, ce qui leur confère, s'ils souhaitent partir au Japon, un statut privilégié. Ce statut, accordé à tous les Péruviens de descendance japonaise de deuxième ou de troisième génération, permet une émigration facilitée, notamment depuis la promulgation en juillet 1990 au Japon d'une loi amendant celle sur l'immigration, datant de 1952. Cette nouvelle loi offre à ces descendants un statut spécifique de « résident» leur permettant de travailler au Japon sans aucune restriction. Pour autant, la réalité apparait plus complexe. Au Japon, les Péruviens se divisent en deux catégories : ceux qui, descendant effectivement des migrants japonais, peuvent rester dans le pays pour y vivre et y travailler, et ceux qui n'ont pas d'ascendance japonaise et qui, très vite, se retrouvent sans papiers, dans l'obligation de travailler de manière illégale et de rester dans la précarité. De nombreux Péruviens ont ainsi tenté leur chance dans ce pays, y compris en recourant à des opérations chirurgicales pour se faire « brider » les yeux. À partir des années 2000, les Péruviens se tourneront vers l'Espagne et l'Italie.

La démocratie au Pérou reste jusqu'à aujourd'hui fragile. Comme le souligne Francisco Belaunde, « la politique péruvienne demeure plus que jamais l'affaire de figures individuelles et non plus de partis ${ }^{29} »$. Et cet auteur de poursuivre sur « la persistance d'une certaine attirance pour les solutions autoritaires chez un nombre non négligeable de Péruviens ». Pour autant, sans doute parce que l'économie du Pérou a toujours su conserver quelques ressorts, notamment avec l'industrie minière, l'immigration des Péruviens n'a jamais atteint, en terme quantitatif, celle de ses voisins équatoriens. On peut donc analyser cette migration des Péruviens en Europe comme mêlant des causes économiques tout autant que politiques. Teofilo Altamarino inscrit le départ de ses concitoyens dans un faisceau relevant de trois causes principales : socioéconomiques, politiques et culturelles.

${ }^{29}$ BELAUNDE, F. (2007), "Pérou : une démocratie sans parti politique", Cahiers des Amériques Latines, $\mathrm{n}^{\circ} 56$, p. 8 . 
Ce chercheur est le premier à évoquer le " retour » comme une éventualité des possibles : «Les Péruviens qui ne rencontreront pas le rêve américain, européen et asiatique verront le retour comme une alternative ${ }^{30}$. »Pour ce sociologue, les causes culturelles renvoient à l'imaginaire de toute migration qui pense que « dans les décisions de migrer, les images et les rêves ont une énorme influence ». Enfin, notons que les Péruviens apparaissent comme les plus diplômés des colectivos d'Amérique latine : « les Péruviens, mieux insérés dans des emplois stables que les autres groupes de migrants, résistent sans doute mieux à la crise que les autres (leur taux de chômage est proche de la moyenne espagnole) $)^{31}$. » Pour autant, devant l'importance de la crise, le retour est devenu une alternative possible. À l'absence de travail s'est en effet ajouté d'autres difficultés, notamment pour rembourser les crédits bancaires (pour ceux ayant accédé à la propriété), mais aussi pour renouveler les papiers, ces derniers étant conditionnés à l'effectivité d'un emploi. Pour les Latino-Américains - Équatoriens, Boliviens ou Péruviens - les possibilités de « choix » sont extrêmement minces et se résument à quelques options : attendre des jours meilleurs en Espagne et affronter la précarité, rentrer dans son pays d'origine ou émigrer dans un pays tiers. Comme le soulignent Virginie Baby-Collin et Geneviève Cortes, « toutes ces options impliquent des tensions fortes dans un processus de décision complet, à la fois individuel et collectif $^{32} »$. La plupart des Latino-américains ont donc choisi de rester, avec des difficultés supplémentaires se traduisant pour eux par des augmentations de la charge de travail, mais aussi par davantage d'activité « au noir ». Enfin, ceux qui avaient acheté une maison se tournent vers la location en essayant de solder leur crédit immobilier.

\subsection{Le difficile retour des Péruviens}

Avant d'analyser les difficultés des Péruviens qui sont rentrés, regardons les réponses institutionnelles. Ces dernières peuvent s'analyser du côté du pays d'accueil - l'Espagne - comme du pays de départ. Concernant l'Espagne, la solution a consisté à réduire de manière drastique les arrivées et à initier une politique de retour volontaire. Les premiers résultats de cette politique sont devenus probants en 2013, avec une baisse sensible des colectivos d'Équatoriens et de Péruviens, comme le montre le tableau $n^{\circ} 3$ :

\footnotetext{
${ }^{30}$ ALTAMIRANO, T. (1999), Los peruanos en el exterior y su revinculación con el Perú, en ligne, http:/www.cholonautas.edu.pe/modulo/upload/Altamirano1.pdf [Consulté le 2.02.2016].

${ }^{31}$ BABY-COLLIN V. et al., 2009, p. 50.

${ }^{32}$ BABY-COLLIN V. et al., 2014, p. 63.
} 
Tableau 3 : Comparaison des résidents équatoriens et péruviens en Espagne durant les années 2000-2013

\begin{tabular}{|l|l|l|l|l|l|}
\hline & 2000 & 2004 & 2008 & 2011 & 2013 \\
\hline Équatoriens & 20481 & 475698 & 427718 & 247360 & 248324 \\
\hline Péruviens & 27422 & 68646 & 121932 & 133309 & 111268 \\
\hline
\end{tabular}

Source : D’après le tableau présenté par F. T. Pérez (Torrez Pérez, 2014, p. 224).

Cette baisse est sans doute à nuancer, comme le précise F. T. Pérez, car « entre 2002 et 2012, il a été attribué la nationalité espagnole à 781318 étrangers résidents en Espagne, et $78 \%$ d'entre eux sont latino-américains ${ }^{33}$ ». Pour autant, si cette baisse apparaît toute relative, les départs d'Espagne demeurent une réalité palpable. Ce même auteur reprend une étude réalisée par l’Université de la Coruña qui montre que « entre 2007 et 2011, 1226000 personnes ont abandonné l'Espagne, et que parmi elles $97 \%$ sont étrangères » (Ibid., pp. 225-226).

Mais revenons à cette politique d'aide au retour de manière plus concrète. Sonia Parella et al. proposent une définition du retour volontaire tel qu'il a tenté de se mettre en place en Espagne : " par "retour volontaire assisté", on entend la mesure d'assistance pour les personnes demandeuses de cet accueil $^{34}$. »

L'Espagne a ainsi mis en place trois types d'aides au retour qui ont fonctionné entre 2009 et 2012 :

- le Programme de retour volontaire concernant les immigrés en situation de vulnérabilité (PREVIE) : 9910 personnes ont été concernées, parmi lesquelles 2192 Boliviens, le « collectif » le plus nombreux ; viennent ensuite les Argentins et les Brésiliens, les Péruviens n’ayant quasiment pas bénéficiés de ces aides.

- le programme APRE qui permet de payer de manière anticipée les prestations chômage aux personnes ayant pu cotiser à la sécurité sociale et souhaitant retourner dans leur pays : 10299 individus y ont souscrit. Là encore, l'immense majorité des bénéficiaires sont des LatinoAméricains. Sonia Parella et al. font l'hypothèse que si ce programme a relativement bien marché (par rapport aux autres), c'est « probablement par les intenses campagnes publicitaires effectuées par le Ministère du travail et de l'immigration $»$.

\footnotetext{
${ }^{33}$ PARELLA S. et al., op. cit., p. 224.

${ }^{34}$ Ibid., p. 173.
} 
- Enfin, le programme de retour volontaire productif, dirigé vers les immigrés qui ont un projet de création d'entreprise associé au retour : 298 personnes en ont bénéficié au total, une quantité plus que résiduelle.

Tableau 4 : Résidents étrangers en Espagne accueillis dans les programmes de retour du gouvernement espagnol (2009-2012)

\begin{tabular}{|l|l|l|l|l|}
\hline & $\begin{array}{l}\text { Programme de } \\
\text { Atención social } \\
\text { (PREVIE) }\end{array}$ & $\begin{array}{l}\text { Programme } \\
\text { «APRE » }\end{array}$ & $\begin{array}{l}\text { Programme } \\
\text { «Productivo » }\end{array}$ & Total \\
\hline Total & 9910 & 10299 & 298 & 20507 \\
\hline Dont Am. Lat. & 9363 & 10250 & 262 & 19875 \\
\hline Dont Argentins & 1412 & 1094 & 3 & 2509 \\
\hline Dont Boliviens & 2192 & 88 & 70 & 2350 \\
\hline Dont Brésiliens & 1384 & 656 & $/$ & 2040 \\
\hline Dont Equatoriens & 958 & 4608 & 68 & 5634 \\
\hline Dont Péruviens & 213 & 749 & 31 & 993 \\
\hline
\end{tabular}

Source : D’après le tableau de F.T. Pérez (Torrez Pérez, 2014, p. 227).

Si l'on additionne l'ensemble de ces trois programmes, c'est un peu plus de 20000 personnes qui ont pu y prétendre ; cela est extrêmement faible, voire anecdotique, au regard de ce qu'imaginait le gouvernement espagnol. À ces plans mis en œuvre par l'État, il faut rajouter d'autres possibilités d'aides développées par les ONG, également très confidentielles. Enfin, notons que les migrations dans un pays tiers sont restées, elles aussi, extrêmement minoritaires. Cette dernière remarque relativise, là encore, le discours sociologique évoqué précédemment et vantant, souvent de manière excessive, toutes les formes de mobilité.

L'échec des aides au retour développées par le gouvernement espagnol montre que les différents «plans de retour » proposés n'ont jamais véritablement fonctionné. En France, ce plan a été concocté par Lionel Stoléru, sous la présidence de Valéry Giscard d'Estaing, en juin 1977. Il aurait néanmoins permis, selon les statistiques officielles de la Direction de la Population et des Migrations, au 31 décembre 1981 (soit quatre ans et demi après son instauration), à de 94000 personnes de bénéficier de cette aide au retour ${ }^{35}$. Il apparaît évident de proposer autre chose aux immigrés qu'un simple dédommagement financier, et ce, quelque soit la discussion autour du montant de ce dernier. Les personnes arrivées avec moult difficultés dans un autre pays, en y ayant de surcroît travaillé pendant de

${ }^{35}$ Selon la revue du GISTI, Plein droit, n4, juillet 1988. 
longues années, attendent mieux qu'une seule aide pécuniaire. Il s'agirait de bâtir de véritables projets de codéveloppement impliquant une réelle concertation entre les pays de départ et le pays d'accueil. Il est donc compréhensible que dans l'état actuel des choses, « les étrangers préfèrent attendre et "contourner" la crise qu'ils estiment temporaire ${ }^{36} »$.

Du côté des pays d'accueil, s'est mis en place une conférence sud-américaine des migrations (CSM) à laquelle participent la plupart des pays d'Amérique du Sud. Il s'agit principalement de réfléchir à l'immigration interne, importante sur le continent. En 2010, la CSM approuva un Plan sud-américain des migrations qui se centra « sur le développement humain de la personne migrante ${ }^{37} »$. Ce plan visait à préconiser des règles de bonne conduite, telle que, par exemple, le fait « de ne pas criminaliser le migrant en fonction de sa situation administrative, de ne pas utiliser le migrant comme une variable d'ajustement de l'économie ou du marché du travail » (Ibid.). Il faut relire cette phrase en supprimant les négations pour comprendre comment le capitalisme financier a très largement contribué à transformer progressivement le migrant en simple variable d'ajustement de l'économie ainsi qu'à le " criminaliser » en fonction de sa situation administrative. Les aides plus spécifiques en matière d'aide au retour des personnes présentes en Europe se sont aussi mises en place en Amérique Latine, mais n'ont pas pu produire les effets escomptés faute de moyens suffisants. Le Pérou, dès le milieu des années 2000, avait commencé à réfléchir à des moyens pour accompagner ces retours volontaires. La loi dite « de réinsertion économique et sociale pour le migrant de retour ", publiée début 2013, a permis d'exonérer de taxes les Péruviens revenant au pays avec leurs meubles, leur voiture mais aussi leurs outils de travail ${ }^{38}$. Les résultats ne semblent pas très probants jusqu'à aujourd'hui, même si aucune étude précise n'a été menée sur ces personnes. D'après nos premiers entretiens, cette loi a permis au moins aux Péruviens de revenir avec leurs affaires personnelles, y compris avec leur outil de travail dans le cadre de la création d'une activité en Espagne, sans être imposés sur ces marchandises.

Elida, rencontrée dans la ville minière de Cerro de Pasco, en témoigne :

\footnotetext{
36 « Los extranjeros prefieren quedarse y « capear » el temporal de la crisis ». Ningún Inmigrante se « apunta » al plan para retornar a su país. Diaro Jaén, Miercoles 24 de diciembre de 2008. Voir également un article de La Croix du 9 septembre 2009, « Les immigrés ne quittent pas l'Espagne».

${ }^{37}$ SASSONE, S. et YÉPEZ DEL CASTILLO, I., 2014, p. 30

${ }^{38}$ Cette loi n 30001 du 14 mars 2013 (« Ley de reinserción económica y social para el migrante retornado ») permet aux Péruviens de retour (à la seule condition d'avoir résidé quatre ans à l'extérieur du pays de manière ininterrompue) d'être libérés en une seule fois du paiement des taxes sur les biens suivants : meubles (jusqu'à concurrence de 30.000 dollars US), un véhicule (jusqu'à 30.000 dollars US) et les machines de travail (jusqu'à 150000 dollars US).
} 
« Il y avait des gens qui avaient une petite entreprise, par exemple d'électricité... ils avaient leurs propres outils d'électricien... Eh bien, ils ont rapporté tous leurs outils et ont été exonérés d'impôts (en rentrant au Pérou)...

- L'exonération durant un an?

Isabel : Non, toute la vie ! Oui, c'est la loi qui s'appelle la loi de acogimiento, la loi du retour pour les Péruviens...

- Et elle est toujours valable?

Isabel : Oui, elle est toujours en cours. Autant que je sache, elle est toujours en vigueur! Mais l'année dernière, elle a été plus forte encore, parce que beaucoup de gens d'Espagne sont revenus... Principalement des ouvriers, des personnes travaillant dans les maisons [des Espagnols comme domestiques] et les travailleurs indépendants qui avaient un petit atelier de réparation et tout... Voilà principalement, parce que les affaires ont diminué, et donc les gens qui travaillaient dans une entreprise sont devenus chômeurs. »

Une enquête réalisée auprès de leurs voisins boliviens par Sonia Parella et al. montre que « seulement $12,5 \%$ d'entre eux répondent affirmativement à la question "considérez-vous que les migrants boliviens qui veulent rentrer en Bolivie reçoivent suffisamment d'aide de la part du gouvernement bolivien pour réintégrer leur pays ?"39 ». Et les auteurs de conclure que leur analyse sur les programmes de retour volontaires impulsées depuis l'Espagne « met en évidence l'insuffisante coopération avec les pays d'origine ». Finalement, ces aides au retour ne seraient qu'une mesure - une parmi d'autres - envisagée par les pays occidentaux pour contrôler leur immigration. Cependant, comme les tentatives de fermeture des frontières, ces mesures n'ont pas montré jusque-là de résultats patents. Et quid des programmes de codéveloppement, sensés donner aux potentiels candidats au départ des opportunités pour rester dans leur pays ? Leur résultats ne seraient guère meilleurs si l'on en croit Stéphane Tapia : « cette logique d'éloignement du territoire, lorsque l'immigration est jugée indésirable se combine avec un autre logique, dont l'efficacité est tout autant relative : celle de l'aide au développement, du co-développement, de la solidarité internationale ${ }^{40}$. » Et cet auteur de poursuivre qu' « entre le cynisme économique et la générosité humaniste (parfois humanitaire), les limites sur le terrain du développement économique durable sont bien difficiles à tracer » $(i d$.$) .$

Devant la faiblesse des réponses institutionnelles, on s'aperçoit que l'acte de retour reste une décision éminemment complexe et personnelle. Comme le

\footnotetext{
${ }^{39}$ Ibid., p. 184.

${ }^{40}$ Tapia, 2007, p. 92.
} 
précisent encore Sonia Parella Rubio et al., «c'est une décision que prennent les immigrés eux-mêmes en fonction de différents éléments en relation avec les circonstances personnelles et familiales, mais aussi avec le contexte économique, social et politique, à l'échelle locale et nationale ${ }^{41} \gg$. Beaucoup de Péruviens sont donc retournés par leur propre moyens, en marge de tous ces dispositifs institutionnels, sans doute parce que la contrepartie de ces programmes s'avérait extrêmement élevée : renoncer aux «papiers » espagnols et s'engager à ne pas revenir en Espagne pendant au moins trois ans. Ceux qui retournent constituent donc une infime minorité, on l'a souligné, la plupart des Latino-américains ayant préféré rester en Espagne pour affronter la crise, avec des stratégies multiples et variées, mais jamais simples.

Cela n'a pas rendu notre enquête facile, y compris lorsque nous nous sommes rendus au Pérou durant l'été 2014 pour tenter de rencontrer ceux et celles ayant vécu cette « expérience du retour ». Nous avons privilégié les rencontres à Lima, mais aussi à Trujillo (ville côtière du Nord du Pérou d'où de nombreux Péruviens sont partis), sans occulter les hauts plateaux andins (autour de la ville minière de Cerro de Pasco). Nous avons plutôt cherché à développer, dans un premier temps, un petit réseau de connaissances susceptibles de nous mettre en contact par la suite avec des personnes de leur entourage ayant tenté ce retour. Nous avons pu rencontrer une dizaine de Péruviens revenus d'Espagne et une Péruvienne revenue récemment du Japon.

Nous souhaitons revenir ici sur le parcours d'Isabel, que nous avons pu rencontrer à Lima, en Août 2014. Cette Péruvienne est arrivée en Espagne bien avant les années 2000, cela lui conférant un regard à la fois aigu et distancié sur le phénomène migratoire de ces dernières décennies en Espagne :

«C'est vrai que quand je suis arrivée, en 1992, il n'y avait pas beaucoup d'étrangers en Espagne !

- Il n'y avait pas beaucoup d'étrangers ?

Isabel : Non, non... et c'était bizarre... parce que quand vous arrivez dans un pays, vous essayez de trouver quelqu'un qui vous ressemble, qui parle péruvien comme vous... Mais je n'en ai pas rencontrés! Alors vous regardez... et puis la façon de s'habiller aussi... je portais des vêtements d'ici (du Pérou) mais en Espagne, ils sont différents ! Alors quand vous descendez dans la rue, vous vous sentez différent parce que vous êtes habillée différemment et les gens vous regardent ! Mais ça, c'était le cas à ce moment-là, mais plus aujourd'hui parce qu'après, l'Espagne a reçu beaucoup de gens (d'immigrés) ! Mais dans ces années-là, il y avait peu d'étrangers et l'Espagne ne s'était pas habitué à recevoir des étrangers...

${ }^{41}$ PARELLA, S. et et al., op. cit., p. 172. 
car normalement en Espagne, c'étaient les Espagnols qui partaient (dans un autre pays)!

- Oui oui, bien sûr ...

Isabel : L'Espagne ne recevait pas de personnes...

- Il y avait seulement quelques Allemands, des Français, des Anglais...

Isabel : Oui, mais plutôt dans le Sud, plutôt dans les îles. Mais il n'y avait pas de Sud-Américains ! Mais après, plus tard, il y a eu beaucoup de gens qui sont venus d'Équateur et du Pérou. »

Isabel est arrivée en Espagne comme étudiante pour poursuivre des études en tourisme, puis a trouvé un travail dans ce domaine et s'est mariée avec un Espagnol. Isabel a même demandé - et obtenu - la nationalité espagnole :

« Comme j'aime voyager, durant les deux premières années que j'étais en Espagne, quand j'ai vu que j'allais rester davantage, j'ai demandé la nationalité... mais seule, moi toute seule, pour moi-même...

- Seule?

Isabel : Oui, parce que quand je voyageais [par exemple] en Italie, je devais demander un visa... Et si j'étais espagnole, ils ne me demanderaient plus de visa pour aller n'importe où... comme je voulais voyager à travers l'Europe avant de venir, je me suis dit que j'allais demander la double nationalité... parce qu'ainsi, je serai tranquille avec mes papiers et tout le reste...

- Et ça été facile de l'obtenir?

Isabel : Et bien oui... bon, quand je les ai demandés, ils ne me les ont pas donné tout de suite, mais cela a pris deux ans... À l'époque, il n'y a avait pas beaucoup de demandes (un temps de réflexion)... non, il a fallu un an, et puis après (pour les autres personnes) il faudra deux ans, voire encore plus... J'avais présenté tous les documents qu'ils m'avaient demandés, et finalement j'ai eu la nationalité ! Et alors, je pouvais voyager où je voulais (rires)! Et puis quand je me suis mariée, j'étais déjà espagnole, alors je n'ai eu aucun problème avec les papiers... Mais il y avait des gens qui se sont mariés...

- Pour obtenir les papiers... ?

Isabel : Oui, pour obtenir les papiers, mais peu de gens, vraiment... Oui, peu, parce que le gouvernement avait facilité les choses pour que les gens puissent avoir les papiers en règle... parce qu'ils vous disaient “À l'endroit où vous avez travaillé, il n'y a rien à faire : juste que la personne (espagnole) dise que vous avez travaillé avec elle, et voilà !’. La régularisation a été bonne parce que la plupart des gens n'ont pas eu besoin, ni de payer, ni de se marier ou d'autres choses comme ça pour les obtenir. » 
Suite à un divorce douloureux et à la maladie de son père (les médecins péruviens lui ayant pronostiqué quelques mois d'espérance de vie), Isabel, désormais seule et sans enfant, décide de rentrer à Lima :

« J'avais divorcé, et le divorce a été très dur, très dur... Et je suis retournée à Madrid pour travailler et j'ai vécu seule... et j'ai su que mon père, on lui avait diagnostiqué un cancer... Et donc, j'ai décidé de retourner vivre avec eux, avec ma maman et mon papa...

- D'accord... Donc la décision de retourner au Pérou, c'était le divorce et la maladie de votre père... Ceux sont deux éléments...?

Isabel : Oui... j'étais seule... seule, et la maladie de mon père, ce sont les deux causes déterminantes qui m'ont décidé à revenir. »

Son retour fut extrêmement difficile, notamment pour se réadapter à la vie de la capitale péruvienne, Lima restant une ville chère pour se loger, bruyante, polluée et à la circulation difficile. Ce retour a été moins douloureux grâce à l'aide d'une ancienne amie de jeunesse, qui lui a permis, après quelque temps, de retrouver un emploi dans le tourisme à Lima, mais avec des conditions beaucoup moins favorables que celles qu'elle avait en Espagne. Son salaire, peu élevé, l'a contraint à loger dans un quartier populaire d'habitat collectif, Los Olivos, loin du centre-ville et peu sécurisé en soirée. Le fait de s'être fait voler dans son propre pays, la perturbe énormément :

« Ici, il n'y a aucune facilité pour vivre (un temps de réflexion)... Il n’y en a pas... Il y a beaucoup d'insécurité... J'ai déjà été volé (depuis mon retour)... j'ai été volé dans un taxi !

- C'est vrai ?

Isabel : Ben oui... Je ne pensais pas que je pouvais être volée ici... Pour moi, quand je suis arrivée en Espagne... je suis arrivée en 1992, et quatre ans après, j'ai été volé à Madrid... Mais une seule fois : en 21 ans, je n'ai été volée qu'une seule fois !

- En 21 ans...

Isabel : Oui, je n'ai été volé qu'une seule fois... (et pourtant) je sortais (la soir), je conduisais, et tout le reste! Mais ici, il n'y un qu'an que je suis arrivée et on m'a déjà volé une fois. Et maintenant, je ne peux plus utiliser le téléphone mobile (dans la rue) parce qu'on te l'arrache... Lorsque vous êtes dans le taxi, vous devez serrer votre sac très fort... c'est très dangereux... Et le trafic automobile est très désordonné. »

Isabel a donc le sentiment de vivre un déclassement social et professionnel important, ce d'autant plus qu'elle voir tous les jours, pour y travailler, les contrastes entre les beaux quartiers du centre-ville et les autres : 
« Je vis à Los Olivos, dans le coin nord de Lima... Mais je travaille à Miraflores $^{42}$. C'est clair, Miraflores, c'est un autre monde ! San Isidro, Miraflores, ce sont des autres mondes... C'est vrai que quand vous êtes à Miraflores, pour moi c'est comme si vous étiez à Madrid! Je suis tranquille, je sors mon téléphone, je parle (au téléphone), en regardant juste un peu (autour de moi)...

- Mais pour louer un logement à Miraflores...?

Isabel : Louer un petit logement dans Miraflores, c'est cher, très cher ! Je me suis renseignée pour vivre près de mon travail, mais une chambre, c'est près de 800 Soles [soit environ 200 Euros]... et c'est le moins cher, parce que ça peut être aussi 1000 Soles ! Et puis les chambres sont la plupart du temps pour les étudiants ou les travailleurs. »

Notons enfin que de nombreux Péruviens sont revenus du Japon ces dernières années, après avoir compris que la mobilité sociale tant attendue ne viendrait plus dans ce pays où ils restaient cantonnés à des tâches déqualifiées et sans perspective d'évolution. Nous avons pu nous entretenir avec Marí, une femme de 35 ans venant de rentrer du Japon à Lima. Marí confirme ce que Karsten Paerregaard écrit : « Une dizaine d'année après que l'émigration des nikkeijin au Japon a commencé, la majorité des Péruviens continuent d'avoir peu d'interaction avec la société japonaise, d'un part à cause des barrières linguistiques et culturelles, d'autre part à cause de leur position très marginale sur le marché du travail. Comme les Péruviens sont souvent embauchés par des intermédiaires, ils perdent leur emploi du jour au lendemain. Cela signifie, une fois de plus, qu'ils doivent rester en perpétuel mouvement pour rechercher et trouver d'autres contrats de travail ${ }^{43}$. »

Au terme de cette enquête, nous avons montré que les migrations de retour constituaient un nouveau sujet d'étude pour la sociologie de l'immigration. Ces dernières accompagnent une mondialisation et un capitalisme qui exigent une flexibilité toujours plus accrue de la force de travail. Ces migrations de retour marquent une instrumentalisation croissante de la main d'œuvre étrangère et dépeignent par défaut des politiques publiques qui ne peuvent plus, sur la durée, intégrer les nouveaux venus. De tels scénarii, s'ils étaient amenés à se poursuivre, conduiraient à la fin de l'intégration (qui se réalise d'abord et avant tout par le travail) et à un délitement du « vivre-ensemble».

Concernant le profil des retornados péruviens, il semble que ce ne soient ni les plus vulnérables, ni les plus « installés » qui retournent, mais ceux qui,

\footnotetext{
${ }^{42}$ Miraflores est une peu le quartier « Saint-Michel » de Lima ; c'est un des plus animé et des plus agréable à vivre de la capitale.
}

${ }^{43}$ PAERREGAARD, K., op. cit., p. 90. 
conscients de leur précarité, ont encore la possibilité d'agir. Si on peut rapprocher nos premières conclusions de l'étude menée sur le retour des Boliviens par Parella et al., il semblerait que ce soit davantage les femmes qui retournent que les hommes, notamment pour s'occuper de leurs enfants restés au pays ou de leurs parents qui vieillissent. Cette idée, selon laquelle les femmes se retrouveraient davantage concernées que les hommes par la notion du care reste cependant à approfondir et à développer.

\section{RÉFÉRENCES BIBLIOGRAPHIQUES}

ALTAMIRANO, T. (1985). "Migración de retorno en los andes". Cuadernos de Investigación del INANDEP, Lima: PISDAL/ INANDEP, $\mathrm{n}^{\circ}$ 2, Enero, pp. 8-41.

ALTAMIRANO, T. (2009). Migración, remesas y desarrollo en tiempos de crisis. Lima: PUCP-CISEPA.

ANSION J. (2008). "Emigrantes peruanos : Cuando los hijos se quedan". In Godard, H. et Sandoral, G. (eds.), Migración transnacional de los Andes a Europa y Estados Unidos. Actes et Mémoires de l'IEFA, tome n 17, IFEA/PIEP/IRD, pp. 211230, Julio.

BABY-COLLIN, V. et CORTÈS, G. (2014). "Nuevos despliegues del campo migratorio boliviano frente a la crisis". In Sassone, S. et Yépez des Castillo, I. (coord.), Migración y crisis global. Europa-America latine: nuevas estrategias, nuevas desigualdades. Revista CIDOB d'Afers Internacionals, ${ }^{\circ}$ 106-107, septembre, pp. 61-83.

BLASQUEZ VILAPLANA, B. (coord.) (2013). Actas del seminario intencional: Retorno de los/as inmigrantes a sus paises de procedencia u origen. Estado de la cuestión. Universidad de Jaén, Colección Actas.

CASTEL, R. (1995). Les métamorphoses de la question sociale. Paris: Seuil.

COHEN, A., CAPOTE A., PUMARES, P., JOLIVET, D., CARVAJAL, C. y DE MIGUEL, V. (2011). "Inmigrantes marroquíes en municipios de Andalucía (2006-2008): entre permanencia e inestabilidad". In Isabel PUJADAS et al. (eds.), Población y Espacios urbanos. Barcelona: Departamento de Geografía Humana de la UB y Grupo de Población de la AGE.

DIRECTIVE 2008/115/CE DU PARLEMENT EUROPÉEN ET DU CONSEIL du 16 décembre 2008 relative aux normes et procédures communes applicables dans les États membres au retour des ressortissants de pays tiers en séjour irrégulier. 
ESCRIVA CHORDA, Á. (1997). "Control, composition and character of new migration to south-west Europe: The case of Peruvian women in Barcelona". Oxford: New Community (devenu Journal of Ethnic and Migration Studies), Vol. 23, Issue 1, pp. 43-57.

ESCRIVA CHORDA, Á. (2003). “Inmigrantes peruanas en España. Conquistando el espacio laboral extradoméstico”. Revista Internacional de Sociología, Vol. 61, $\mathrm{n}^{\circ} 36$, pp. 59-83.

GERMANA, C., MENESES, M., VALENCIA, I., COLLARON, R. y SAMAMÉ, D. (2005). La migración internacional. El caso peruano. Lima: Fondo Editorial de la Facultad de Ciencias Sociales (UNMSM).

GLICK SCHILLER, N., BASCH, L., SZANTON BLANC, C. (1992). "Towards a Transnational Perspective on Migration : Race, Class, Ethnicity, ans Nationalism Reconsidered". Annals of the Nex York Academy of Sciences", n 645, pp. 1-24.

HILY, M.-A. (2004). Critique de la notion de retour. Les ressources d'une approche qualitative. Atelier du CEPED des 5 et 6 avril 2004.

JEDLIKI, F. (2007). “Les retours des enfants de l'exil chilien. L'empreinte du politique dans les parcours d'insertion". In Véronique PETIT (ed), Migrations internationales de retour et pays d'origine. Nogent-sur-Marne: CEPED, pp. 193-205.

LAFFORT, B. (2009). “Le parcours des Maliens en Andalousie (Espagne), où comment l'agriculture espagnole a créée un nouveau prolétariat”. NAQD, revue algérienne d'études et de critique sociale. $\mathrm{N}^{\circ}$ spécial Migrants, Migrance, El harga. $\mathrm{N}^{\circ}$ 26/27, Automne/hiver, pp. 303-333.

LAFFORT, B. (2010). "Quand la dernière campagne de ramassage des olives en Andalousie (2008/09) révèle un drame plus profond: celui des immigrés qui travaillent dans l'agriculture". In Aïssa KADRI, Gérard PRÉVOST y Gloria ESTEBAN DE LA ROSA (sous la dir. de), Inmigración e integración de los inmigrantes desde una perspectiva hispano-francesa en el contexto de las actuales politicas comunitarias sobre inmigración. Granada: Ed. Comares, pp. 371-412.

LAFFORT, B. (2012). “Amargas aceitunas españolas”. In Gloria ESTEBAN DE LA ROSA y José Alfonso MENOR TORIBIO (sous la dir. de), Estudios e informes sobre la inmigración extranjera en la provincia de Jaén 2009-2011. Granada: Ed. Comares, pp. 460-495.

LEY DE REINSERCION ECONOMICA Y SOCIAL PARA EL MIGRANTE RETORNADO (JUEVES 14 DE MARZO DE 2013). LEY N 30001.

MA MUNG, E., DORAI, M. K., HILY, M.-A. et LOYER, F. (1998). "La circulation migratoire. Bilan des travaux". Migrations Études, $\mathrm{n}^{\circ} 84$.

MATEO, A. M. y QUEIRELO PAMAS, L. (2005). “Migrar desde las Américas a Europa. Jóvenes, familias y subjetividades en las nuevas migraciones internacionales", 
Alternativas $n^{\circ} 13$, Cuaderno de trabajo social. Alicante: Escuela universitaria de trabajo social de Alicante y Universidad de Alicante.

MICHALON, B. (2007). "Les retours en migration : une notion polysémique, des formes migratoires multiples". In Migrations internationales de retour et pays d'origine, op. cit., pp. 27-45.

REVUE EUROPÉENNE DES MIGRATIONS INTERNATIONALES (2013). Migrations de retour et de rapatriement. Vol. 29, $\mathrm{n}^{\circ} 3$.

SASSONE, S. y YÉPEZ DEL CASTILLO, I. (coord.) 2014. "Migración y crisis global. Europa-America latine: nuevas estrategias, nuevas desigualdades". Revista CIDOB d'Afers Internacionals. $\mathrm{N}^{\circ} 106-107$, septiembre.

SAYAD, A. (1999). La Double absence. Des illusions aux souffrances de l'immigré. Paris: Le Seuil, 1999.

SAYAD, A. (1991). L'immigration ou les paradoxes de l'altérité. Bruxelles: Éditions universitaires/De Boeck.

TAKENAKA, A. (1999). "Transnational Community and Its Ethnic Consequences. The Return Migration and the Transformation of Ethnicity of Japanese Peruvians". American Behavioral Scientist 42. June, pp. 1459-1474.

TAKENAKA, A., PAERREGAARD, K. (2012). "How Contexts of Reception Matter : Comparing Peruvian Migrants' Economic Trajectories in Japan and the US". International Migration, published on line, 29 Nov.

(DE) TAPIA, S. (2007). “Du projet intégré de développement économique à l'invisibilité: les retours dans l'émigration turque". In Véronique PETIT, Migrations internationales de retour..., op. cit., pp. 87-110.

TARRUIS, A. (2000). Les nouveaux cosmopolitismes. Mobilités, identités, territoires. La Tour d'Aigues: Éditions de L'Aube.

TARRIUS, A. (2001). “Au-delà des États-nations : des sociétés de migrants”. Revue européenne des migrations internationales. Vol. 17, $\mathrm{n}^{\circ} 2$, pp. 37-61.

TORIBIO MENOR, J. A. (2010). “Características de la inmigración en España y análisis de su integración a través de algunos indicadores". In Aïssa KADRI, Gérard PRÉVOST y Gloria ESTEBAN DE LA ROSA (sous la dir. de), Inmigración e integración de los inmigrantes..., op. cit., pp.163-189.

VIANA BARBOSSA, L. (2013). "A dificil desisao do retorno trabalhadoras domesticas bolivianas e peruanas em tempos de cirse economica, Seminarion internacional". Fazendo Gênero 10 - Desafios Atuais dos Feminismos, Brazil, Florianopolis: Universidade Federal de Santa Catarina, 16-20 de setembro. 
